\title{
Integriertes Hochwasserrisikomanagement in Österreich
}

Mit dem Hochwasser 2002 kam es in Österreich nach einer Phase fehlender großräumiger, katastrophaler Ereignisse seit 1965/66 zu einer Zäsur. Stand davor der Hochwasserschutz im Vordergrund, mit dem Ziel „hochwasserfrei“ zu werden, so zeigte sich 2002 (aber auch 2005), dass es Grenzen gibt und ein hundertprozentiger Schutz nicht möglich ist. Die detaillierten Analysen der Hochwässer 2002 und 2005 erfolgten in den beiden Projekten FloodRisk I und FloodRisk II. Die praktischen Erfahrungen und Ergebnisse dieser Projekte, aber auch Erkenntnisse aus den Extremereignissen in anderen Ländern der EU in diesem Zeitraum ergaben den Handlungsbedarf auf europäischer Ebene.

Unter österreichischer EU-Ratspräsidentschaft kam es 2006 zur Vorbereitung der EU-Hochwasserrichtlinie, die 2007 in Kraft trat. Mit diesem Meilenstein und der nationalen Implementierung in der Novelle 2011 des Wasserrechtsgesetzes kann man den Übergang vom Hochwasserschutz zum Integrierten Hochwasserrisikomanagement in Österreich verbinden. In der Folge und unter Berücksichtigung der Empfehlungen aus den FloodRisk-Projekten gab es in allen Bereichen signifikante Weiterentwicklungen, die von rechtlichen Änderungen bis zu Optimierungen technischer Maßnahmen reichen. Anlässlich des Hochwasserereignisses 2013 startete das Projekt FloodRisk_E(valuierung) mit dem Ziel, die erfolgten Verbesserungen zu analysieren und den noch bestehenden Handlungsbedarf festzustellen.

Dieses Heft der Österreichischen Wasser- und Abfallwirtschaft beleuchtet in acht Beiträgen einzelne Themen des Integrierten Hochwasserrisikomanagements in Österreich, wobei klar ist, dass diese nur einen Ausschnitt dieser umfassenden und komplexen Materie behandeln.

Im ersten Artikel „FloodRisk_E(valuierung)“ analysieren Habersack et al. die erzielten Verbesserungen und den verbleibenden Handlungsbedarf im Integrierten Hochwasserrisikomanagement in Österreich und beurteilen die Umsetzung der Empfehlungen aus FloodRisk I sowie FloodRisk II unter Berücksichtigung der EU-Hochwasserrichtlinie. Es zeigte sich, dass insgesamt bedeutende Fortschritte erzielt wurden und sich die institutionelle Zusammenarbeit in gemeinsamen integralen Projekten verbessert hat. Gleichzeitig besteht noch Untersuchungs- und Handlungsbedarf.

Die Umsetzung der EU-Hochwasserrichtlinie (RL 2007/60/EG) in Österreich ist das Thema des zweiten Beitrags von Neuhold. Es wird dargestellt, wie die einzelnen Arbeitsschritte der Richtlinie auf Basis bundesweit einheitlicher Methoden in Österreich umgesetzt und international koordiniert wurden. Aufbauend auf den Risikoklassen „hohes“ und „sehr hohes Risiko“ wurden Gebiete mit potenziellem signifikantem Hochwasserrisiko (391 APSFR-Gebiete) ausgewiesen. Es folgt die Beschreibung der Hochwassergefahren- und risikokarten und der Maßnahmenplanung samt Priorisierung und Maßnahmenprogramm.

Hornich et al. behandeln im dritten Artikel das Integrierte Hochwasserrisikomanagement in der Steiermark und Oberösterreich. Noch unter dem Eindruck der Hochwasserereignisse des Jahres 2002 wurde in der Steiermark ein Sachprogramm erarbeitet, welches klare rechtliche Rahmenbedingungen für Baumaßnahmen in Hochwassergebieten geschaffen hat. Die Erstellung der Hochwasserrisikomanagementpläne war ein Beteiligungsprozess mit begleitender Öffentlichkeitsarbeit. In Oberösterreich wird neben der Verbesserung der Prognosesysteme vor allem auf integrale Bearbeitungen bei Gefahrenzonenplänen und im Bereich des Katastrophenschutzes gesetzt.

Als Planungsinstrument für ein integratives Flussraummanagement stellen Mühlmann et al. im Beitrag 4 die Gewässerentwicklungs- und Risikomanagementkonzepte (GE-RM) vor. Der GE-RM Planungsprozess erfolgt für längere Gewässerstrecken bzw. ganze Einzugsgebiete und führt zur Defini- 
tion eines integrativen Leitbilds, welches in weiterer Folge als Grundlage für die Erstellung konkreter schutzwasserwirtschaftlicher und ökologischer Maßnahmenkonzepte dient. Der Leitfaden 2016 soll in den kommenden Jahren in der Praxis erprobt, evaluiert und entsprechend den konkreten Erfahrungen überarbeitet werden.

Artikel 5 von Schober et al. stellt die Floodplain Evaluation Matrix (FEM) als umfassende Methode zur Bewertung von Überflutungsflächen im Rahmen des Integrierten Hochwasserrisikomanagements dar. Der Schutz - und wo möglich die Wiederherstellung - von Überflutungsflächen wird seit Jahren gefordert. Dennoch steht dem in der Realität ein fortschreitender Flächenverbrauch gegenüber. Die FEM stellt eine Methode dar, mit der es möglich wird, den Wert von zusammenhängenden Überflutungsflächen festzustellen und Grenzwerte zu definieren, die eine fortschreitende Verbauung hintanhalten.

Zahnt et al. analysieren in Beitrag 6 die Herausforderungen durch pluviale Überflutungen. Anhand einer durchgeführten Untersuchung in der Steiermark an betroffenen Gebäuden werden die Entstehung pluvialer Überflutung, die damit verbundenen Schäden und die sich ergebenden Konsequenzen beleuchtet. Da pluvialen Hochwässern sowohl unterschiedliche Prozesse zugrunde liegen, als auch verschiedenste Fachbereiche Aufgaben in der Vorsorge, der Bewältigung und der Nachsorge übernehmen, liegt der Schwerpunkt der Bearbeitung auf einem integralen Betrachtungsansatz.

Mit dem Zusammenhang zwischen Hochwässern und Feststoffen beschäftigt sich Artikel 7 von Haimann et al. Sedimente werden im Hochwasserfall z.B. aus Stauräumen remobilisiert, jedoch auch in strömungsberuhigten Bereichen wie Vorländern abgelagert. Anlandungen im Flussprofil können zu früheren Ausuferungen führen. Außerdem erhöhen Sedimente den Schaden und steigern die Kosten für Aufräumarbeiten. Ein Feststoffmanagementkonzept kann helfen, das Sedimentkontinuum wiederherzustellen, und die Berücksichtigung des minimalen flussmorphologischen Raumbedarfs kann das Hochwasserrisiko sowie Schäden minimieren.

Im Artikel 8 beschreiben Klösch et al. die Rolle der Vegetation im Hochwasserabfluss. Im Projekt „VEGSED“ werden in einem $5 \mathrm{~m}$ breiten Forschungsgerinne Versuche im Maßstab 1:1 mit Durchflüssen von bis zu $10 \mathrm{~m}^{3} \mathrm{~s}^{-1}$ und bis zu $3 \mathrm{~m}$ Wassertiefe durchgeführt, wodurch Laboruntersuchungen mit Felduntersuchungen vereint werden. Dieser Artikel behandelt die Wirkung eines Vegetationsverbands auf die Hydrodynamik und den Sedimenttransport sowie die Überprüfung eines Pflanzenverformungsmodells und dessen Kopplung mit einem zweidimensionalen Hydrodynamik- und Sedimenttransportmodell.

Univ.-Prof. DI Dr. H. Habersack (ه)

Department für Wasser - Atmosphäre

- Umwelt, Christian Doppler Labor für

Sedimentforschung und -management, Institut für Wasserwirtschaft,

Hydrologie und konstruktiven

Wasserbau, Universität für Bodenkultur

Wien,

Muthgasse 107,

1190 Wien, Österreich

helmut.habersack@boku.ac.at 